\title{
Section in Ophthalmology of the New York Academy of \\ Medicine
}

\section{Sitzung vom 17. April 1899}

R. Denig zeigt einen Fall von traumatisch em Enophthal-mos infolge eines Revolverschusses. Die Kugel war wie gewòlmlich gegen die recbte Schläfe in selbstmörderischer Absicbt abgefeuert, batte die recbte Orbita quer durchschlagen, den Sehnerven kurz vor seiner Einpflanzung getroffen und zablreiche Aderbaut- und Netzhautblutungen verursacht; sie batte dann ihren Weg durcli das linke Antrum Iligh-mori genommen und blieb schliesslich im linken Processus pterygoideus sitzen, von wo sie extrabiert wurde. Die Lokalisation mit den RöntgenStrablen stiess insofern auf Schwierigkeiten, als Patient wegen $\Lambda$ Volfs-racbens einer Operation unterzogea worden war und eine enorme Schwellung des linken Gaumens und reichlieher Speichelfluss bestand. Denig betont, dass das Röntgenbild Frakturen oder eine Zertrümmerung in der rechten Orbita nicht nachweisen konnte und dass das Auftreten von Enophtbalmos bei der Häufigkeit dieser Art von Revolverschuss-verletzungen in der Litteratur noch nicht verzeicbnet ist. Vielleicht lag eine Verletzung der syrnpathischen Wurzel des Ganglion ciliare vor.

Trigeminusstörungen war en nicht vorhanden.

In der Diskussion sprachen P. Fridenberg, P. A. Callan und E. Gruening.

H. Knapp zeigt einen Patienten, bei dem er die llaab'sche

Magnetoperation vorgenommen hatte. Der Fremdkörper hatte die

Ho.rnhaut und Linse durchschlagen und befand sich im Glaskörper.

Der llaab'sche Magnet wurde 2 Tage nach der Verletzung angewandt

Zeitschrift f. Augenheilkunde. Bd. II. Heft 6. ' $\quad \wedge^{\circ}$

598 Sitzungsberichte.

und förderte den Fremdkórper zu Tage, naehdem nocli ein Hornhaut-schnitt ausgeführt worden war. Die partiell getrübte Linse klärte sich $\lambda$ víeder auf und Patient bekam normale Sehschärfe. In der Diskussion sprachen Th. R. Pooley, E. Gruening, Fridenberg und Callan. Gruening bemerkt, dass es ziemlich häufig sei, dass die partielle Cataract, die bei Glaucomoperationen durch Verletzung der Linse entstünde, sich wieder aufkläre. Knapp erwähnt, dass er die Methode, den Fremdkörper zum hintern Pol der Linse zu ziehen und dann uin ihre Peripherie herumzuleiten, 6 mal ohne Erfolg versucht habe.

J. Wolff demonstriert 2 Fälle von einseitiger angeborener Ab-ducenslährnung mit Retractionsund Protrusionsbewegungen der Augen. Die Fälle sind analog dem Türk'scken Falle. Wolff nimmt mit Turk an, dass der Rectus externus durch einen unnachgiebigen sehnigen Strang ersetzt ist. Es kann deshalb das xluge nicht nach aussen be-wegt werden. $\Lambda$ Venn das Auge versucht, sich nach innen zu drehen, so hindert dieser Strang den Rectus int. an der Ausübung seiner Funktion, und es entsteht dann ein Zug von hinten an beiden Seiten des Bulbus, am Ansatz des unnachgiebigen Stranges und des Rect. int., so dass das Auge nur nach hinten nachgeben kann. T. R. Chambers zeigt einen Fall, in dem er wegen Entfernung eines Tumors im inneren Canthus eine Plastik ausgeführt hatte. 
E. Gruening berichtet über eine sympathische Ophthalmie nach einer Ilornhautverletzung. Er betont, dass eine reine Corneal- $\Lambda$ Vunde mit ausgedehntem Irisprolaps unter konservativer Behandlung eine sympathische Ophthalmie hervorrufen kann. Iiöchst wahrscheinlich ware diese in seinem Fall durch rechtzeitige Excision der vorgefallenen Iris verhütet worden.

Die anatomische üntersuchung des enukleirten Auges zeigte, dass die incarcerierte Iris die Ciliarfortsätze in die Länge gezogen, ab-geflacht und in die vordere Kammer gezogen batten. W. H. Bates verliest eine Abhandlung über den Gebrauch des ГTebennierenextrakts bei Augenkrankheiten (wird in den Archives of Ophthalmology veröffentlicht werden). Sitzung vom 15. Mai 1899.

H. Knapp zeigt 5 Bulbi, die nach subchorioidalen Blutungeu enukliert worden waren, und zwar waren die Blutungen aufgetreten nach Starextraktionen, nach Iridocyclitis, Glaucom und Bulbusluxation.

J. Guttmann zeigt einen Fall von Intraorbital-Abscess infolge Empyem des Antrum Highmori durch cariöse Zähne.

Knapp glaubt, dass der Abscess vom Frontalsinus ausging und Pooley und Johnson, dass es sich um ein Sarcom des Antrum handeln könne.

T. R. Pooley berichtet über einen Fall von Herpes zoster ophthalmicus. $1 \alpha$ der Diskussion erwähnt Leszynsky einen Fall, den er beobachtete, und bemerkt, dass die Erkrankung verursacht wurde durch eine akute degenerative Neuritis der Supraorbital-N erven, wo bei die Analgesie und Neuralgie über ein Jahr anhielt. Pooley glaubt an

Therapeutische Urns chau. 599 eine Entzimdung des Nerven in seinen Zweigen oder im Ganglion Gasseri.

Chas. H. May zeigt einen Fall von Augenverletzung infolge eines Nagels.

T. R. Poo ley einen solcben von van Fleet's Modifikation der Pan as' sclien Ptosis-Operation.

F. Valk verliest eine Abhandlung über Strabismus. (Aus den Archives of Ophthalmology).

Denig-New York.

Ophthalmological society of the United Kingdom.

Oct. 12. 1899. (The Brit. med. Journ.)

G. Anderson Britchett gab einen kurzen historischen Ueber-blick über die Entwicklung der

Cataract-Extraction von den ersten Ver-suchen Daviel's im Jahres 1745 bis auf unsere Tage.

Lawford berichtete über 5 Fälle von interstitieller Keratitis bei erworbener Syphilis. Er meinte, dass bei dieser Form der interstitiellen Keratitis die einzelnen Fälle von kürzerer Dauer seien als bei der auf hereditärer Lues beruhenden, dass meistens nur ein Auge befallen werde und die Prognose im allgemeinen güustiger zu stellen sei.

Harviau society of London. Octob. 19. 1899.

Juler stellte ein 7 jähr. Kind mit Microphthalnius conge.nitus des rechten Auges vor. Ferner stellte er einen 37 jährigen Patienten vor, bei dem sich eine Parese beider äusseren

Augenmuskeln, Retinal-blutungen und schwankender Gang gezeigt hatte, welcher eine intracranielle Erkrankung vortäuschte. Der Patient war Alcoholiker und litt an chronischer Nephritis. "Der weitere Verlauf der Erkrankung, be-sonders das fast vollständige Zurückgehen der Augenmuskellähmung bewies, dass es sich um ein organisches Gehirnleiden nicht handeln konnte. Es wird deshalb angenommen, dass die Lähmung durch toxische Ein $\Lambda$ virkung Alcoholismus oder üraemie - entstanden sei.

Silex.

Therapeutische Umschau. 
Behandlung des Glaucoma chronicum simplex mit Galvanisierung des Hals-Sympathikus, Dr. Allard. (La clinique ophthalm.

No. 20

18 99.) Als Пeílmittel gegen Glaucoma chronicum simplex ist in neuester Zeit bekanntlich die Resektion des $\Gamma$ lals-Sympathikus mehrfach 